\section{Transvaginal urethral diverticulectomy using small intestinal submucosa interposition graft}

\author{
Karen De Baets, Ann M.A. De Vylder \\ Jeroen Bosch Ziekenhuis, Department \\ of Urology, 's Hertogenbosch, \\ The Netherlands
}

\section{Abstract}

Background. Our aim was to evaluate our four and a half year single-center experience with interposition of small intestinal submucosa (SIS) in transvaginal urethral diverticulectomy.

Design and methods. We reviewed the records of all patients who underwent a transvaginal diverticulectomy at our center between September 2006 and December 2010. Collected data included demographic details, symptoms, diagnosis, treatment, follow-up, and outcome. Mean time of follow-up was 127 days.

Results. Twelve consecutive patients were identified. Mean age was 36 years. Most reported symptoms were a vaginal mass and pain, recurrent urinary tract infections, and urinary stress incontinence. At physical examination a vaginal mass was palpated in all cases. In two out of seven patients cystourethroscopy showed the diverticulum. Magnetic resonance imaging was diagnostic in five out of ten patients who underwent this examination. In all patients a transvaginal diverticulectomy with SIS interposition was performed. In one patient the postoperative course was complicated by a minor postoperative bleeding that needed re-intervention. Two patients developed de novo urinary stress incontinence after surgery. No symptoms of diverticulum recurrence, urethrovaginal fistula, urethral stricture or voiding difficulties were reported.

Conclusions. We conclude that SIS is efficient and safe to use as interposition graft in transvaginal urethral diverticulectomy.

\section{Introduction}

Female urethral diverticula (UD) are a rare urological entity. Lately the incidence is increasing due to a greater awareness and better imaging methods. In most cases diagnostic delay exists, this shows that many urologists and gynaecologists are still not familiar enough with UD. Another contributing factor to this delay is the variable clinical presentation and the fact that the symptoms are often only intermittently present. Various nonspecific symptoms are reported, i.e. dysuria, dyspareunia, post-void dribbling, recurrent urinary tract infections (UTI), urethral discharge, frequency, and urgency. Diagnosis is often made by detailed history-taking and thorough clinical examination. Because of its detailed anatomical description, magnetic resonance imaging (MRI) is considered the most accurate imaging method. ${ }^{1-4}$ Nevertheless, voiding cystourethrography (VCUG), cystourethroscopy, and transvaginal ultrasound are also used as a diagnostic tool. The treatment modality of choice is transvaginal diverticulectomy. ${ }^{1-3,5,6}$ In some publications the use of an autologous tissue interposition graft is described, i.e. a Martius fat pad, ${ }^{1,3,5}$ bulbospongiosus muscle, ${ }^{1}$ and vaginal flap. ${ }^{3,7}$ Small intestinal submucosa (SIS) is an acellular biologic graft harvested from porcine small intestinal submucosa. It is commercially available (Surgisis ${ }^{\circledR}$, Cook Biotech) and approved for human use. The treatment of Peyronie's disease, correction of hypospadias, correction of severe penile chordee, and penile reconstructions are some of the urological applications in which it is since long time applied. ${ }^{8-12}$ In this publication we report on four and a half year single-center experience in transvaginal diverticulectomy with interposition of SIS and review the recent literature.

\section{Design and Methods}

We performed a retrospective review of the medical records of all patients who underwent a transvaginal urethral diverticulectomy at our center between September 2006 and December 2010. The following data were collected: demographic details, date of first presentation, symptoms, duration of symptoms, previous treatment, findings on physical examination, date and method of diagnosis, date and details of surgical repair, pathology report, duration of follow-up, and outcome.

UD was defined as a localized outpouching in the anterior vaginal wall connected with the urethra through a usual narrow diverticular neck.

All patients are operated by the same reconstructive urologist. The patient is placed in dorsal lithotomy position. First generation cefalosporines and metronidazole profylaxis is provided intravenously. The labia majora are spread and a transurethral catheter (14-Fr Foley catheter) is inserted. An inverted U-shaped incision is made in the anterior vaginal wall over the diverticulum (Figure 1). The diverticulum is dissected from the surrounding tissue and communication with the urethra is identi-
Correspondence: Karen De Baets, Jeroen Bosch Ziekenhuis, Department of Urology

Postbus 90153, 5200 ME 's Hertogenbosch, The Netherlands.

Tel. +31.73.5532434 - Fax: +31.73 .5532373 .

E-mail: debaetskaren@gmail.com

Key words: reconstructive urology, small intestinal submucosa, urethra, urethral diverticulum.

Contributions: KDB, conception and design, acquisition, analysis and interpretation of data, manuscript drafting, administrative, technical or material support; ADV, conception and design, data analysis and interpretation, manuscript critical revision, supervision.

Conflict of interest: the authors report no conflicts of interest.

Received for publication: 31 August 2011. Accepted for publication: 11 0ctober 2011.

This work is licensed under a Creative Commons Attribution NonCommercial 3.0 License (CC BYNC 3.0).

(OCopyright K. De Baets and A.M.A De Vylder, 2011

Licensee PAGEPress, Italy

Urogynaecologia 2011; 25:e14

doi:10.4081/uij.2011.e14

fied (Figure 2). After resection of the diverticulum, the urethral lesion is closed over the catheter with polyglactin sutures (Figure 3). Subsequently, the SIS graft is tailored, moistened, and placed in the space between the urethral and the vaginal wall (Figure 4). Next, the anterior vaginal wall is closed over the biomaterial. Finally, a vaginal tampon with estrogen gel is inserted during $24 \mathrm{~h}$. Antibiotic profylaxis is maintained during $48 \mathrm{~h}$ whereupon the patient is discharged with the catheter in situ. Except for two patients, all underwent a VCUG before removing the catheter approximately ten to fourteen days postoperative.

\section{Results}

Twelve patients underwent transvaginal urethral diverticulectomy at our center between September 2006 and December 2010. Mean age was 36 years (range 21-54 years). Seven patients (58\%) presented with a combination of a urethral/vaginal mass and discomfort. Five patients (42\%) complained of recurrent UTI, four (33\%) of urinary stress incontinence (SI). Three patients (25\%) presented with urethral loss of old blood or pus and two patients (17\%) had additional complaints of dysuria, frequency and urgency. Mean duration of the symptoms was 21 months (range 1-85 months). At vaginal exami- 
nation a mass in the anterior vaginal wall was palpated in all patients. In five cases meatal loss of pus and urine occurred when palpating the mass. Seven patients underwent a cystourethroscopy. In two patients this exam revealed the diverticular orifice, in the five others no abnormalities were found. Ten patients underwent a MRI of the pelvis. In half the patients (5/10), the test was diagnostic and anatomical details were described. In two patients the lesion was described as a paraurethral cyst (Figure 5), and in two of the remaining patients it was unclear whether a connection with the urethra existed. Finally, in one patient no abnormalities were seen because the diverticulum drained spontaneously a few days before the MRI. The mean diameter of the diverticula was $2.4 \mathrm{~cm}$ (range 1-3 cm). The mean time between the first visit and diverticulum diagnosis was 30 days (range 0-94 days). In all patients a transvaginal diverticulectomy was performed by the same reconstructive urologist. SIS biomaterial was used as interposition graft between the urethral and vaginal wall. One patient developed a postoperative bleeding that needed revision the same day. During the reoperation the SIS graft was removed and a haemostatic patch $\left(\mathrm{TachoSil}^{\circledR}\right)$ was used. No new SIS graft was inserted. Further postoperative course was uneventful. The mean duration of transurethral catheterisation was 12 days (range 2-22 days). Ten patients underwent a VCUG before removal of the catheter. In all but one case the pathology report confirmed the diagnosis of UD. In one patient only fibrotic tissue was found microscopically. This finding correlates with her history of multiple severe UTIs before diverticulectomy. The mean time of follow-up after diverticulectomy was 127 days (range 10-539 days). No complaints of urethrovaginal fistula, urethral stricture or voiding difficulties were reported; neither symptomatic diverticulum persistence or recurrence. Two patients (16.7\%) developed de novo urinary SI (Table 1). In these patients an expectative approach was followed but since persistence of the incontinence and no benefit from physiotherapy, a suburethral sling procedure was planned in these patients. In all four patients with pre-existing SI, the incontinence resolved after diverticulectomy. One patient no longer had SI but complained of transient urgency postoperative (Table 1). Anticholinergics were administered with good results.

\section{Discussion}

UD is a rare urological problem with various clinical presentations. It is not surprising that diagnosis is often delayed because of the wide range of subtle and transient symptoms. In the series presented here the most reported symptom was perineal discomfort due to a urethral
Table 1. Pre- and postoperative symptoms.

\begin{tabular}{lcc} 
Symptoms & $\begin{array}{c}\text { Preoperative } \\
\text { no. of patients }(\%)\end{array}$ & $\begin{array}{c}\text { Postoperative } \\
\text { no. of patients (\%) }\end{array}$ \\
Urethral/vaginal mass/discomfort & $7(58.3)$ & 0 \\
Recurrent UTI & $5(41.7)$ & 0 \\
\hline Urinary SI & $4(33.3)$ & $2(16.7)$ \\
Loss of old blood/pus & $3(25)$ & 0 \\
\hline Dysuria & $2(16.7)$ & $1(8.3)$ \\
Frequency/urgency & $2(16.7)$ & 0 \\
\hline
\end{tabular}

UTI, urinary tract infection; SI, stress incontinence.

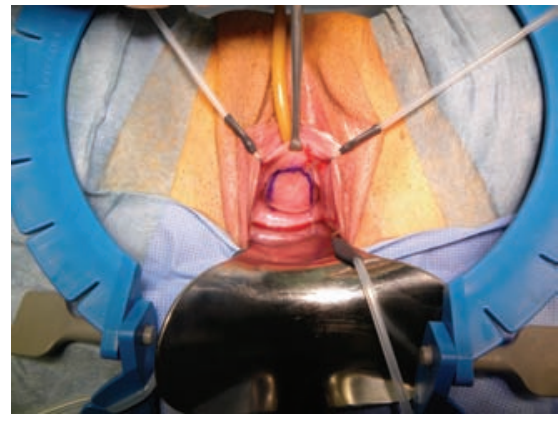

Figure 1. An inverted U-shaped incision is made in the anterior vaginal wall over the diverticulum.

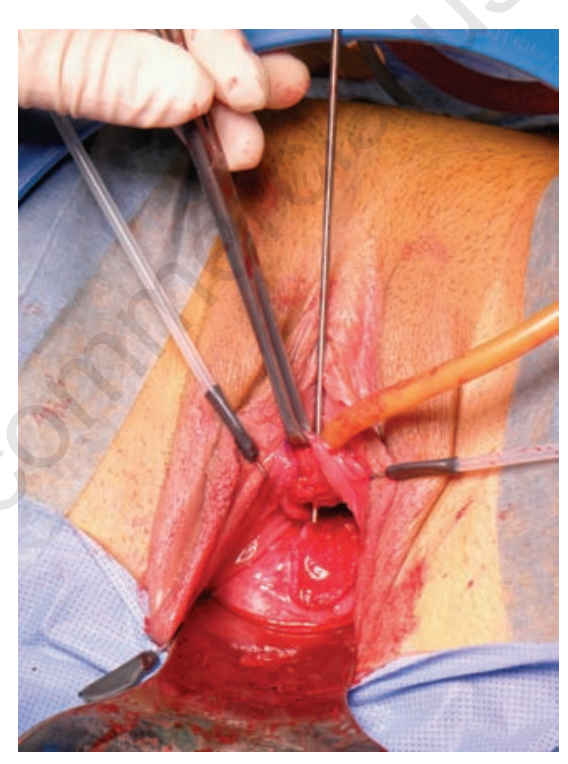

Figure 2. The diverticulum is dissected and resected and communication with the urethra is identified.

or vaginal mass, followed by recurrent UTI and urinary SI. The mean interval between the first presentation and the diagnosis was 30 days (range 0-94 days) which is rather short in comparison to other studies. ${ }^{13,14}$

In all patients in our series the UD was palpable as a vaginal mass. In other publications this percentage was not so high. ${ }^{1-3,5,13,15}$ In only $42 \%$ pus or urine emerged from the meatus by palpation of the UD. Although pathognomonic, this sign is only found in a minority. ${ }^{2,3,5,14} \mathrm{~A}$

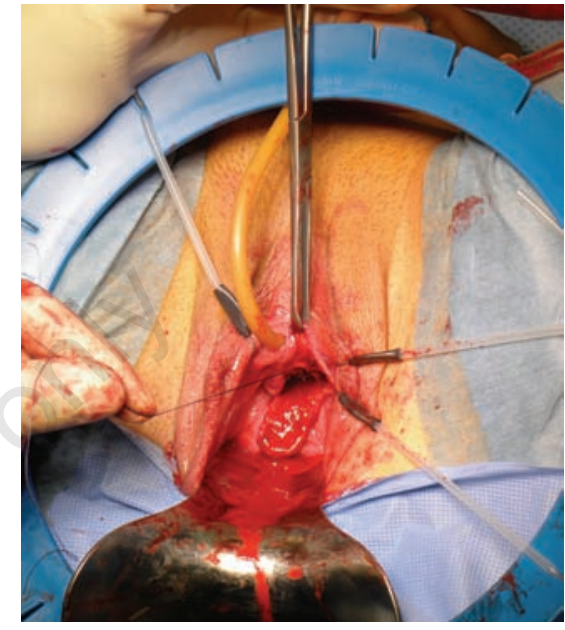

Figure 3. The urethral lesion is closed over the catheter with polyglactin sutures.

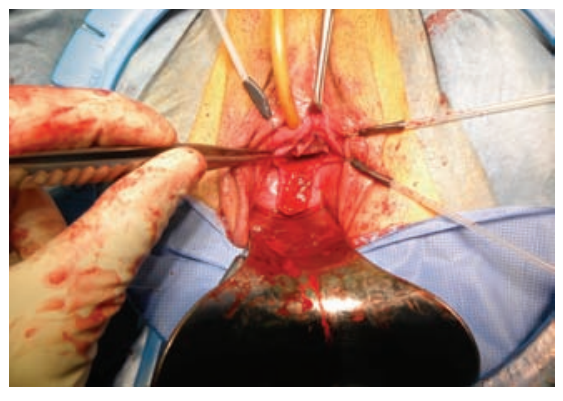

Figure 4. The small intestinal submucosa graft is tailored and placed in the space between the urethral and the vaginal wall.

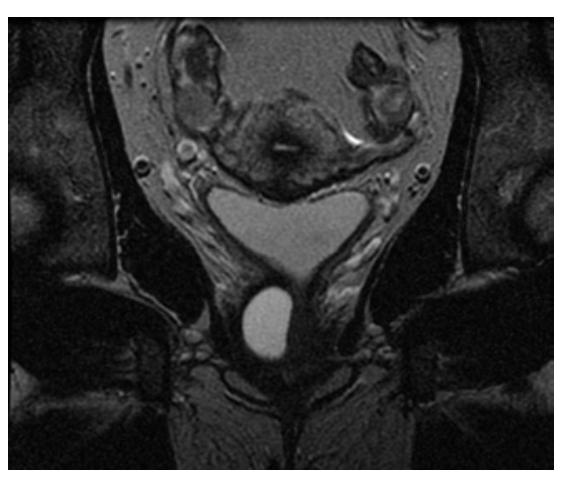

Figure 5. Urethral diverticulum described as a paraurethral cyst on magnetic resonance imaging. 
possible explanation for this low percentage can be a very narrow communication between the UD and the urethra or the fact that the UD is not filled with urine during physical examination. ${ }^{14}$

To confirm the diagnosis of UD a MRI of the pelvis was performed in ten patients. Because of its detailed anatomical description of the diverticular sack and its relation to the surrounding tissue many authors consider MRI as the imaging method of choice. ${ }^{1-4}$ Despite this, the diverticular neck is infrequently visualized on MRI. In a recent publication of Chung et al. MRI had an error rate of $24.4 \%{ }^{4}$ One of their explanations for the diagnostic errors was that the small connection between the UD and the urethra may not always be filled with fluid, making it difficult to detect. Another reason is the fluctuating size of UD. When the diverticulum just drained it may be too small for MRI sensitivity or may not be filled with liquid and may not be visualised therefore. ${ }^{4}$ In our series the error rate was even higher (50\%). In many centers surface and endorectal coils are used with improved sensitivity.

Different surgical techniques are described to treat UD. Spence and Duckett described the technique of extensive marsupialisation that resulted in an elongated urethrovaginal fistula. ${ }^{16}$ Diverticulum recurrence, vaginal voiding, and spraying of urine with micturition are further complications of this procedure. ${ }^{17}$ TurnerWarwick and Chapple favor performing a transvaginal diverticulectomy with immediate sphincter reconstruction, as they state that all UD are associated with a local sphincter deficiency and, consequently, an inherent intrinsic sphincter weakness even if the patient is continent. ${ }^{18}$

Other previously described techniques are endoscopic incision or resection, ${ }^{19}$ incision and packing of the diverticular cavity ${ }^{20}$ or periurethral injection of Polytef paste adjacent to the diverticulum. ${ }^{21}$ Since most diverticula contain infected material, there exists a considerable risk of infection with these synthetic materials. ${ }^{17}$ Nevertheless, nowadays the standard treatment modality is surgical excision: transvaginal diverticulectomy with or without interposition of tissue between the vaginal and urethral wall. ${ }^{1-3,5,6,22,23}$ Multiple authors describe the use of a Martius fat pad, ${ }^{1,3,5}$ bulbospongiosus muscle ${ }^{1}$ or a vaginal flap ${ }^{3,7}$ as interposition graft. In our center, we started to use SIS interposition in transvaginal diverticulectomy in 2006. This because we were never able to find the distinct peri-urethral tissue layer, in contrast to the surgical descriptions which describe the importance of this layer to prevent against complications such as urethrovaginal fistula. SIS is easy to handle and place because it can be cut, rolled or folded to accommodate the clinical requirements. This makes its use less time-consuming than harvesting an autologous tissue interposition graft. That there are no associated comorbidities from harvesting techniques, is an additional advantage. Moreover, the fact that there is no persistent foreign material makes it very safe to use.

In case of preoperative urinary SI one may consider concomitant anti-incontinence procedures at diverticulectomy. Different opinions are available in literature. Some authors performed simultaneous sling procedures with good results, ${ }^{3,5,22,24}$ while others prefer to delay this procedure until the tissue is healed and primary outcome can be evaluated. ${ }^{1,6,7,13}$ When delayed, it can be reevaluated whether additional procedures are still necessary since in some patients the urinary leakage named $S I$ preoperatively is rather post-void dribbling associated with the diverticulum. Therfore, we also prefer to delay any incontinence procedures. Another reason to delay additional surgery until reevaluation is that the SIS interposition graft can also act as a tensionless suburethral sling.

In the literature de novo SI after transvaginal diverticulectomy is reported in $1.7-49 \%$ of the cases., ${ }^{5,22,23}$ This great disparity is due to the different definitions and the fact that preoperative urinary leakage and postvoid dribbling may be confused with SI making it very difficult to correctly determine the rate of $d e$ novo postoperative SI. ${ }^{23}$ A recent study of Stav et al. reported an incidence of de novo SI of $16 \%{ }^{5}$ They encountered a size above $3 \mathrm{~cm}$ and a proximal location of the diverticulum to be significantly associated with the development of de novo SI postoperative. ${ }^{5}$ In their series only one patient $(4 \%)$ needed surgical treatment for her incontinence, in the other patients the SI was only mild and not bothersome. ${ }^{5}$ In the series of Ingber et al. and Lee $e t$ al. $10 \%$ needed subsequent anti-incontinence surgery. ${ }^{6,23}$ Although we did not found a relationship with size, in the current series, we found a similar incidence of de novo SI (16.7\%). Contrary to Stav et al., in the current series both patients with de novo SI are planned for suburethral sling procedure because of persistent bothering complaints.

Besides de novo SI, other postoperative complications reported in the literature are persistence or recurrence of the diverticulum, development of urethrovaginal fistula and urethral stricture, persistent irritating voiding symptoms, urethral pain and dyspareunie., $7,14,22,24$ None of our patients complained about these. In many cases postoperative fistula development or recurrent diverticulum leads to reintervention. Because previous procedures increase the amount of surgery-related fibrosis the results of these re-interventions are often disappointing. Moreover Ljunqvist et al. state that repeated surgery is the most important cause for long-term residual symptoms. ${ }^{7}$ This demonstrates that the first operation is the most important one. Because the SIS graft provides an additional strength to the repair, we suggest using a SIS interposition graft during the first intervention to prevent complications and possible reoperations.

Finally, we discuss some limitations of this study. First of all, the series reported in this paper consists of 12 cases. Although many published series in literature include only few patients, this must be considered as rather small population. A second limitation refers to the retrospective nature of the study. The data collection was restricted to a period of four years and the follow-up phase of our series is rather short (mean 130 days). Therefore, we acknowledge that further follow-up is necessary to investigate long-term outcomes. Nevertheless, good initial postoperative results were found.

\section{References}

1. Ockrim JL, Allen DJ, Shah PJ, Greenwell TJ. A tertiary experience of urethral diverticulectomy: diagnosis, imaging and surgical outcomes. BJU Int 2009;103:1550-4.

2. Han DH, Jeong YS, Choo MS, Lee KS. Outcomes of surgery of female urethral diverticula classified using magnetic resonance imaging. Eur Urol 2007;51:1664-70.

3. Romanzi LJ, Groutz A, Blaivas JG. Urethral diverticulum in women: diverse presentations resulting in diagnostic delay and mismanagement. J Urol 2000;164:428-33.

4. Chung DE, Purohit RS, Girshman J, Blaivas JG. Urethral diverticula in women: discrepancies between magnetic resonance imaging and surgical findings. J Urol 2010;183:2265-69.

5. Stav K, Dwyer PL, Rosamilia A, Chao F. Urinary symptoms before and after urethral diverticulectomy - can we predict de novo stress urinary incontinence? J Urol 2008;180:2088-90.

6. Ingber MS, Firoozi F, Vasavada SP. Surgically corrected urethral diverticula: long-term voiding dysfunction and reoperation rates. Urology 2011;77:65-9.

7. Ljungqvist L, Peeker R, Fall M. Female urethral diverticulum: 26-year followup of a large series. J Urol 2007;177:219-24.

8. Lee EW, Shindel AW, Brandes SB. Small intestinal submucosa for patch grafting after plaque incision in the treatment of Peyronie's disease. Int Braz $\mathrm{J}$ Urol 2008;34:191-7. 
9. Knoll LD. Use of Small intestinal submucosa graft for the surgical management of Peyronie's disease. J Urol 2007;178:247478.

10. Leslie JA, Cain MP, Kaefer M. Corporeal grafting for severe hypospadias: a single institution experience with 3 techniques. J Urol 2008;180:1749-52.

11. Elmore JM, Kirsch AJ, Scherz HC. Small intestinal submucosa for corporeal body grafting in severe hypospadias requiring division of the urethral plate. J Urol 2007;178:1698-1701.

12. Hayn MH, Bellinger MF, Schneck FX. Small intestine submucosa as a corporal body graft in the repair of severe chordee. Urology 2009;73:277-9.

13. Rufford J, Cardozo L. Urethral diverticula: a diagnostic dilemma. BJU Int 2004;94: 1044-7.

14. Chang YL, Lin ATL, Chen KK. Presentation of female urethral diverticulum is usually not typical. Urol Int 2008;80:41-5.

15. Davis HJ, Telinde RW. Urethral diverticula: an assay of 121 cases. J Urol 1958;80:34-9.

16. Spence HM, Duckett JW. Diverticulum of the female urethra: clinical aspects and presentation of a simple operative technique for cure. J Urol 1970;104:432-7.

17. Graham SD. Female urethral diverticula. In: TE Keane (ed.) Glenn's urologic surgery, 5th ed., pp. 397-406. Philadelphia, Lippincott Williams and Wilkins, 1998.

18. Turner-Warwick R, Chapple CR. Developmental anomalies of the adult female urethra and the sphincter mechanisms. In: Functional reconstruction of the female urinary tract, pp. 580-586. 0xford, Blackwell Publishing, 2002.

19. Lapides J. Transurethral treatment of diverticula in women. Trans Am Assoc GU Surg 1979;70:135-8.

20. Ellik M. Diverticulum of the female urethra: a new method for ablation. J Urol
957;77:243-4.

21. Mizrahi S, Bitterman W. Transvaginal, periurethral injection of polytetrafluoroethylene (polytef) in the treatment of urethral diverticula. Br J Urol. 1988;62: 280.

22. Porpiglia F, Destefanis P, Fiori C, Fontana D. Preoperative risk factors for surgery female urethral diverticula. Our experience. Urol Int 2002;69:7-11.

23. Lee UJ, Goldman H, Moore C, et al. Rate of de novo stress urinary incontinence after urethral diverticulum repair. Urology 2008;71:849-53.

24. Rovner ES, Wein AJ. Diagnosis and reconstruction of the dorsal or circumferential urethral diverticulum. J Urol 2003;170:826. 